\title{
An audio-tactile display
}

\author{
DEANE B. BLAZIE \\ U.S. Army Human Engineering Laboratory, Aberdeen Proving Ground, Maryland 21005
}

and

T. V. CRANMER

Rehabilitation Division, Kentucky State Department of Education, Frankfort, Kentucky 40601

\begin{abstract}
Several devices have been developed enabling the blind to adapt to digital and visual displays. Most require extensive training and are relatively expensive. The audio-tactile display described in this paper requires very little training and promises to be inexpensive. Prototypes of the display have been developed for a digital stopwatch, a digital clock, and several electronic calculators. Because the device makes use of electrical impulses to communicate with the blind, it also has numerous potential capabilities as a research tool. The device is based on a panel containing columns of braille digits that are made active through a logic circuit interfacing the braille display with a calculator or other device. The subject scans the braille columns and discovers the digits active in each column when a tone is produced simultaneously with the finger contacting the braille digit.
\end{abstract}

The overwhelming public acceptance of electronic calculators has led to an all-out search for an adaptation which best makes information derived from these devices available to blind people. Kelly (Note 1) successfully modified the Hewlett-Packard HP-45 calculator by interfacing it with a solenoid-driven braille display. The Science for the Blind organization (Benham, Note 2) successfully produced a prototype of another device that serially interrogates a calculator and produces a braille printout on paper tape. Other devices available commercially provide verbal feedback on the number or key touched.

The audio-tactile display described here allows a user to randomly access digital information by purely electrical means. A permanent record of performance (including response time, etc.) can be made for those interested in research with the blind or investigations of normal cutaneous perception abilities.

At the heart of the display is a metallic plate with holes in the pattern of the braille digits $0-9$. These digits are arranged in vertical columns, as shown in Figure 1. The number of columns equals the number of digits on the visual display to be read.

Each hole in the metal-faced plate has a small ring of metal removed from around it. Metal pins are pressed into the holes, thus forming the raised braille digits. The digits themselves are thus insulated from the metal material of the surrounding plate. Touching the braille digits causes the fingertip simultaneously to touch the surrounding metal, completing the electrical circuit.

In each column there are nine passive digits and one with an electrical charge. When the charged digit is touched with the finger, an audio alarm is triggered. This informs the subject that the digit he is touching is to be read as an active part of the calculator's display. To read the display, the individual scans each column for a tone and compiles the number, a digit at a time. This arrangement may be likened to a visual display in which a vertical column of digits is lit from behind by a row of lights, only one of which is turned on at a given time. Thus, the observer quickly understands that he is to read only the lighted digits.

The audio-tactile display can be used in a variety of ways. Calculators, stopwatches, and digital test instru-

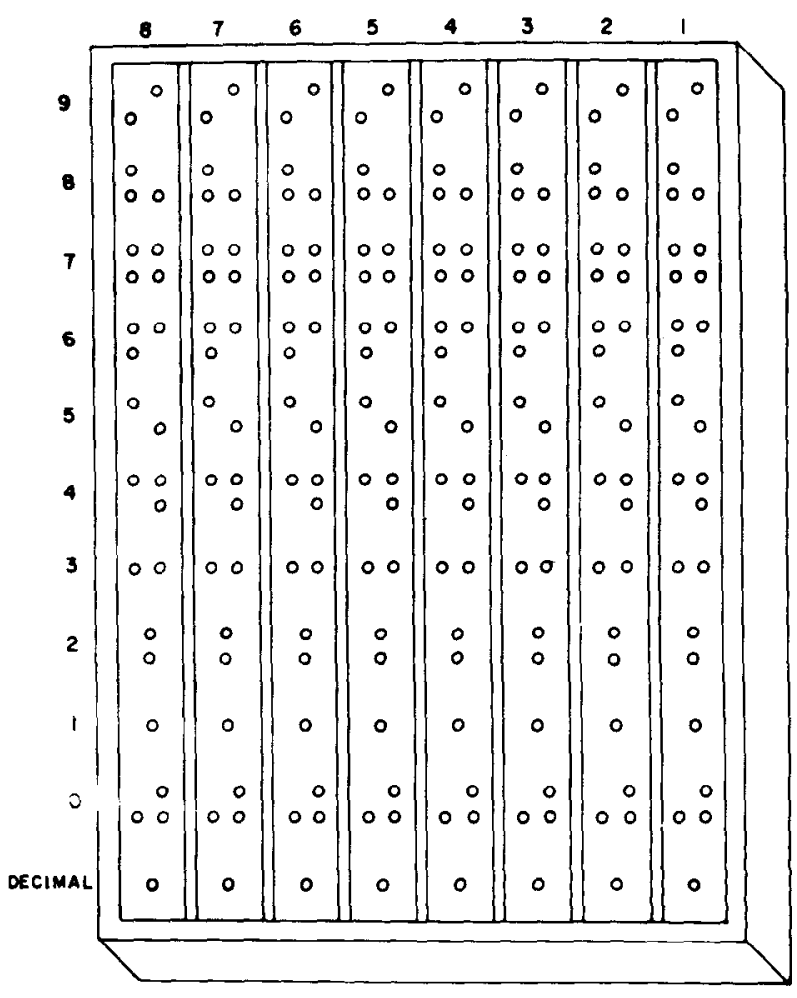

Figure 1. Braille panel for eight-digit calculator. 
ments are the first uses to come to mind; other applications include nonnumeric information displays. For example, a color indicator may display a braille color wheel with dots in a circular fashion. The active dot would indicate the relative color a sensor is responding to.

\section{TECHNICAL DESCRIPTION}

The display is simply a touch-sensitive switch that has been adapted to a braille system. The manner in which the switch "contacts" are used depends upon the use of the display. (Examples will be discussed later.) As noted, the switch operates when two sensory contacts are touched by a finger. Using a $95-\mathrm{mm}$-square plated braille cell and substrate, the skin resistance available to activate the unit can vary from a few megohms for moist fingers to over 50 megohms for very dry fingers. This means that the switch circuit must be sensitive enough to detect a 50 -megohm resistance across the sensor contacts.

The physical display consists of braille cells representing items of information (in the case of a stopwatch, the digits 0-9). A cell is on a conductive substrate such as a printed circuit board (see Figure 2). The raised dots are electrically conductive pins implanted in the pattern of the braille digit and protruding through the board. Each dot is insulated from the conductive substrate by removing the conductive coating in the vicinity of each dot. The dots are electrically tied together on the underside of the display. This point represents one terminal of the switch sensor and the substrate represents the other. These are the sensory contacts mentioned above.

The electronic portion of the switch is shown schematically in Figure 3. It consists of a single CMOS (Complementary MOS) inverter with a pull-up resistor on the input. The input resistance of a typical CMOS inverter is on the order of a million megohms, which makes it ideally suited for our application. The pull-up resistor

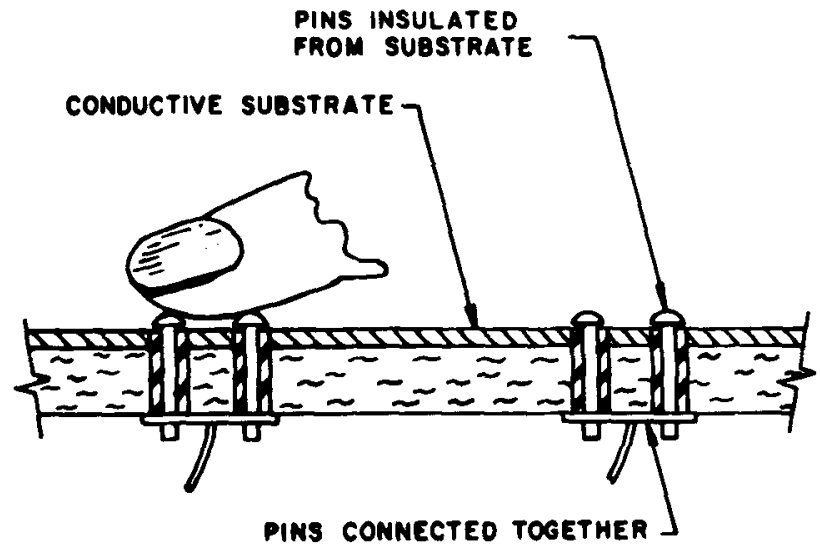

Figure 2. Cross-section of Braille panel.

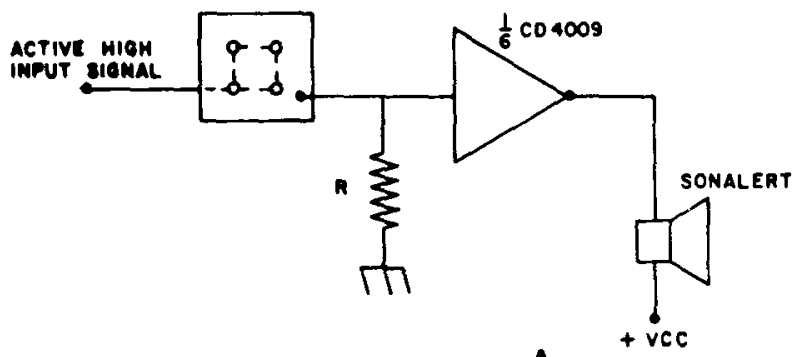

Figure 3. Touch-sensitive switch.

insures the state of the switch when it is not being touched. This resistor may be connected to the positive supply when a logical low signal is being used to activate the display, or it can be connected to ground when a logical high is driving the display. This resistor controls the sensitivity of the touch switch and can be made variable should the need arise to change the sensitivity.

\section{A SIMPLE COUNTER}

Figure 4 shows a decade counter (RCA CD4017 CMOS integrated circuit). The 10 output lines go to the cells on the braille display panel. Each of the 10 output lines on the counter goes to a braille cell corres-

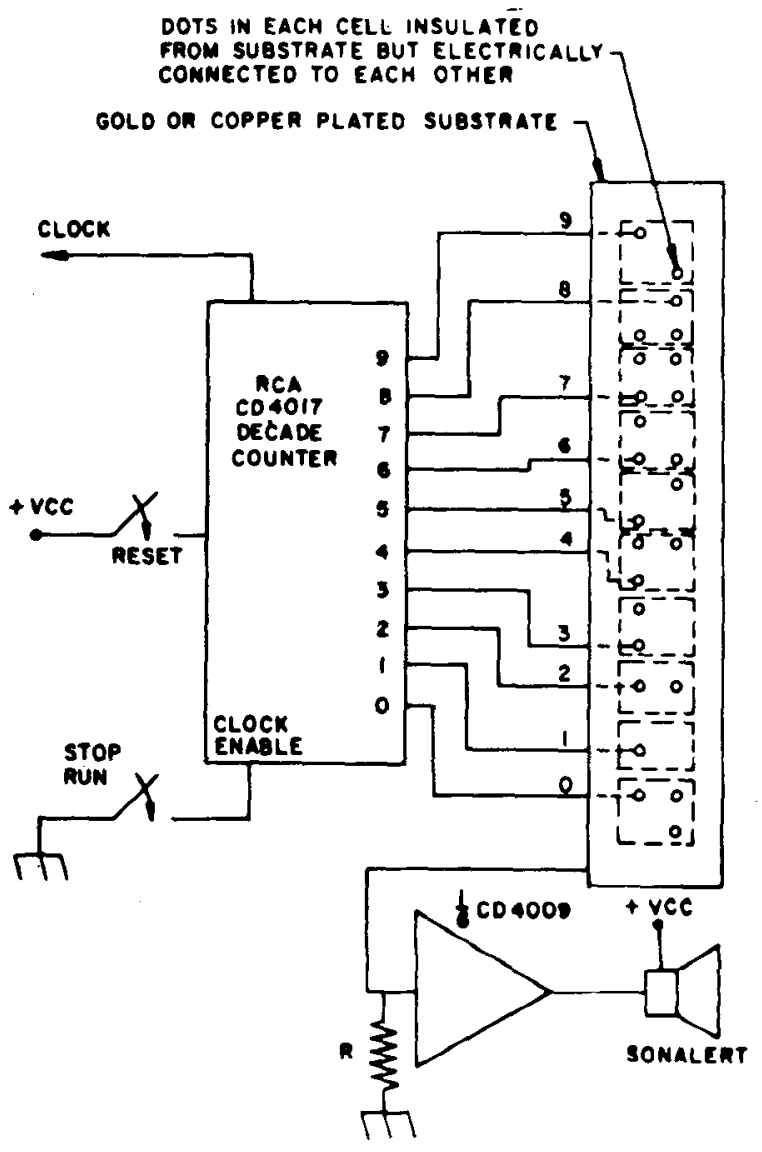

Figure 4. A simple counter. 
Table 1

Parts List for Audio-Tactile Display Single-Digit Counter

$\begin{array}{lll}1 \text { ea } & \text { CD4017 } & \text { Decade counter RCA integrated circuit } \\ 1 \text { ea } & \text { CD4009 } & \text { Hex inverter RCA integrated circuit } \\ 1 \text { ea } & \text { Mallory Sonaler or appropriate alarm } \\ 2 \text { ea } & \text { Single-pole single-throw switch for reset } \\ \text { and run function } \\ 1 \text { ea } \\ \text { Clock source (may be simple switch) } \\ \text { Tactile matrix panel constructed as per } \\ \text { Figure 2 and Figure 6. } \\ \text { 20-M } \Omega \text { resistor }\end{array}$

ponding to the digit on the counter. Referring to Figure 3, each time the count reaches a certain value, 5 , for example, line 5 on the CD4017 goes high; if the braille cell representing Numeral 5 is touched at that time, the substrate causes the inverter to change state and the Sonalert to sound. The alert sounds as long as the cell is touched and the count is 5 . (Table 1 provides the parts list.)

This simple circuit enables one to read a single-digit counter. To display a two-digit counter, simply add another column of braille cells to the substrate and connect the lines from the second counter to the new column of cells. Now a touch on an active cell in either column will produce an audible signal. In this way, any number of digits can be displayed with a single switch circuit.

\section{AN EIGHT-DIGIT CALCULATOR}

Figure 5 is a functional block diagram of a calculator using the audio-tactile display. The Cannon Palmtronic LE-80 calculator incorporates an eight-digit visual display containing an overflow and a minus-sign indicator. The eight digits are made visible by seven-segment LED displays and the minus and overflow indicators by LED dot displays.

The seven-segment to decimal converter changes the calculator's seven-segment display format into a decimal format used on the audio-tactile display. The 10 outputs connect directly to the braille cells on the display panel. The digit-selection logic circuit contains the electronic portion of the touch switch and circuits to handle the demultiplexing and the overflow and minus indication.

The calculator display is time multiplexed, as is

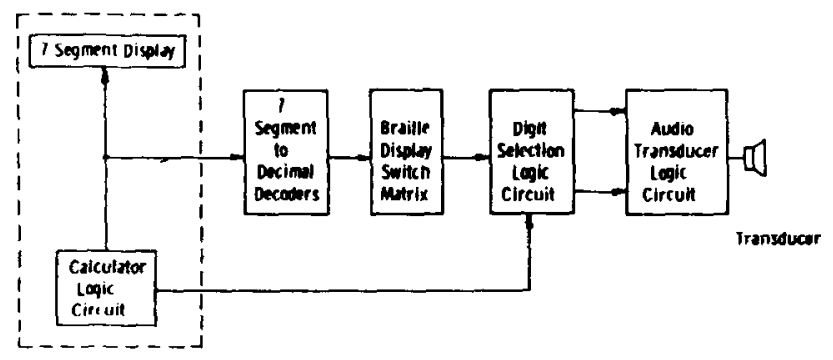

Figure 5. Block diagram of an eight-digit calculator. common with multidigit displays. Each digit is energized for a short time (several microseconds) and is off for a longer time (several milliseconds) while the other digits are being energized. This scheme reduces the number of connections from the calculator to the display but poses a problem to the simple braille circuit just described: Instead of 10 digit lines for each column, we have only 10 lines for all columns. The seven-segment signals must be directed to the proper braille columns at the right instant in time, that is, they must be demultiplexed.

While this could require a considerable amount of logic circuitry, advantage can be taken of the mechanical layout of the braille panel shown in Figure 1. Note the eight columns of braille cells representing the eight digits in the calculator's visual display. Each column contains the digits 9 through 0 from top to bottom, followed by a decimal point at the bottom. The vertical bars between columns represent insulation between the conductive substrate of each column. This breaks up the display electrically into eight separate one-digit (one-column) audio-tactile displays.

Requiring eight touch-sensitive switch circuits may seem to complicate the design, but it actually simplifies the logic required to do the demultiplexing.

The seven-segment to decimal decoder takes as input the seven-segment lines with the multiplexed information for all eight digits. At sequential periods in time, these lines represent the seven-segment code of the eight digits being displayed. Eight digit-select lines, also from the calculator logic circuits, tell at which point in time each digit is to be turned on. Since there is one line for each digit, each time that line is active we can assume the seven segments represent the digit to be displayed.

Gating the eight touch-switch inverters with the corresponding digit-select lines decodes the multiplexed display information. Then we can logically "OR"

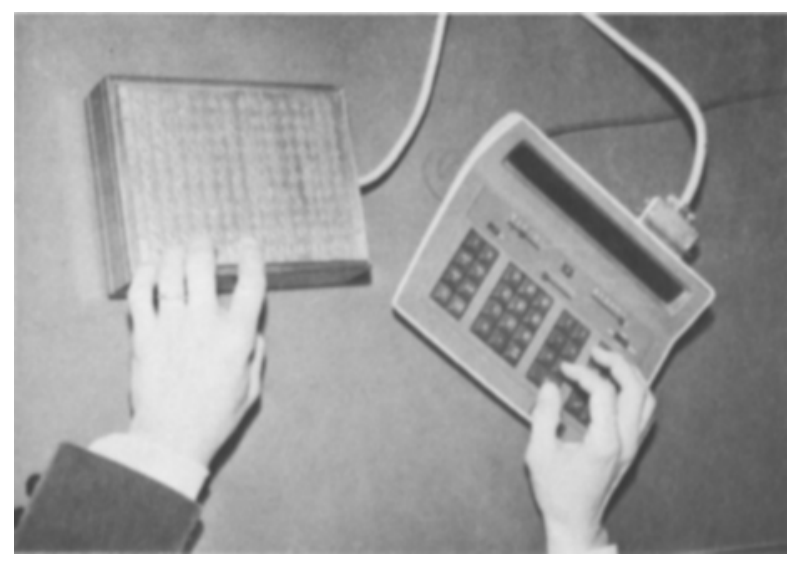

Figure 6. Audio-tactile display incorporated in to a scientific calculator. 
the eight gated column signals to appropriately drive the audio output device.

Although the decimal point is on the display panel, it can be treated exactly as a numeral, except it need not be converted from seven-segment format: There is a separate decimal point signal available in the calculator.

To this point, the sign and overflow indicators have not been mentioned. While separate braille cells can be included to display this information, from a human factors standpoint, it is undesirable. Instead, logic circuits are implemented in the display interface to provide a continuous tone whenever overflow occurs. The tone is activated without touching the display and is sustained until the condition is cleared on the calculator. When a negative number is read, touching an active braille numeral produces a beeping tone instead of the normal continuous tone produced on positive numbers. This scheme requires no operator attention yet keeps the subject informed of the appropriate condition.

\section{SUMMARY}

To date, the audio-tactile display has been successfully used with calculators (see Figure 6), stopwatches, and telephone switch panels. Future plans include digital voltmeters, counters, thermometers, and color indicators. The display is easy to use and requires very little training. It promises to be relatively inexpensive and could be sold with a calculator for under $\$ 200.00$. Although designed for practical use by the blind, the apparatus appears to have potential utility as a research device, not only with the blind but also for studies of tactile communication.

\section{REFERENCE NOTES}

1. Kelly, J. Personal communication, University of New Mexico, Albuquerque, New Mexico, 1974.

2. Benham, T. A. Personal communication, Science for the Blind, Dala Cynwyd, Pennsylvania, 1974.

(Received for publication April 15, 1976; revision raceived July 29,1976 .) 